\title{
Effects of Nutrition Education Interventions in Team Sport Players. A Systematic Review
}

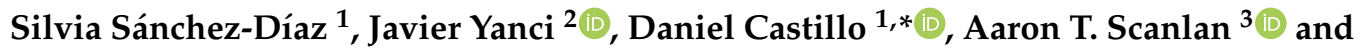 \\ Javier Raya-González ${ }^{1}$ (D) \\ 1 Faculty of Health Sciences, Universidad Isabel I, 09003 Burgos, Spain; silvia.sanchez2467@ui1.es (S.S.-D.); \\ javier.raya@ui1.es (J.R.-G.) \\ 2 Society, Sports and Physical Exercise Research Group (GIKAFIT), Physical Education and Sport Department, \\ Faculty of Education and Sport, University of the Basque Country, UPV/EHU, 01007 Vitoria-Gasteiz, Spain; \\ javier.yanci@ehu.eus \\ 3 Human Exercise and Training Laboratory, Central Queensland University, School of Health, Medical and \\ Applied Sciences, Rockhampton 4702, Australia; A.Scanlan@cqu.edu.au \\ * Correspondence: daniel.castillo@ui1.es
}

Received: 10 November 2020; Accepted: 26 November 2020; Published: 28 November 2020

\begin{abstract}
Considering nutrition education interventions have been frequently implemented in team sport athletes and have shown promising results, this study aimed to summarize the effects of nutrition education interventions on eating habits, nutrition knowledge, body composition, and physical performance in team sport athletes. A systematic review was conducted using the following databases: PubMed/MEDLINE, Web of Science and SPORTDiscus. A total of 14 studies met the inclusion criteria for the review. The methodological quality of included studies was evaluated, and each study was assessed according to the analyzed variables (i.e., eating habits, nutrition knowledge, body composition, and physical performance). Most studies showed improvements in or maintenance of variables used to indicate eating habits, nutrition knowledge, and body composition. However, limited studies examined the effect of nutrition education interventions on physical performance, with existing studies demonstrating disparate results. These findings suggest implementation of nutrition education interventions in team sport athletes could be an effective strategy to improve their eating habits, nutrition knowledge, and body composition. Due to the heterogeneity across the included studies regarding sport modality, competition level, age, and sex of the athletes investigated, as well as the intervention type adopted (i.e., online or face-to-face), it is difficult to establish optimal nutrition education interventions for each analyzed variable.
\end{abstract}

Keywords: nutrition knowledge; eating habits; sports performance; body composition; soccer; basketball

\section{Introduction}

Team sports are played around the world [1], accumulating large audiences [2] and generating high economic impact [3]. In recent decades, the application of sport science has become a staple in most team sports to improve the health and performance of athletes [4]. In this sense, sport science research has grown with most studies focusing on three predominant topic areas including: training and game load monitoring through physical, physiological, and perceptual variables [5]; optimization of athletes' anthropometric characteristics and physical fitness [6]; and evaluation of injury prevention and rehabilitation programs [7]. However, recent research has highlighted the relevance of invisible strategies (i.e., those other than training plans) in the pursuit of optimizing athlete health and performance [8]. Nutrition is considered a key invisible strategy, benefiting performance during competition and facilitating recovery [9]. 
Most team sport studies focusing on athlete nutrition have reported on the health- and performance-related effects of diets [10-12] and supplementation [13-15]. However, some promising data have emerged regarding the efficacy of nutrition education interventions in team sports [16]. Nutrition education interventions are specific programs designed to assist target populations in modifying their eating habits and/or enhancing their nutrition knowledge [17]. These outcomes are particularly relevant for team sport athletes given increasing nutrition knowledge can yield substantial positive changes in eating habits in team sport athletes [16]. In turn, improved eating habits can enhance performance in team sport athletes [18]. Therefore, nutrition education interventions appear to be a key strategy to optimize team sport athletes' performance. Most investigations examining the effectiveness of nutrition education interventions have recruited athletes from individual sports, showing beneficial results in compliance with Mediterranean diet quality index [19] and nutrition knowledge, perceived susceptibility to the Female Athlete Triad, and self-efficacy constructs in specific contexts for each athlete [20]. However, research exploring the application of nutrition education interventions in team sports is more limited than in individual sports, since teams are made up of 15-22 players who have different nutritional needs [21]. Nevertheless nutrition education interventions are being increasingly applied among team sport athletes since interventions can be implemented using a wide range of modalities, including personal interviews [22], group activities [23], comics [24], interactive workshops [23], or technological platforms [25]. However, the effectiveness of each specific modality in nutrition education interventions needs to be analyzed to identify the most appropriate approach relevant to the specific context applied.

Since nutrition education interventions in team sports can benefit athlete nutrition knowledge and dietary behaviors, and in turn performance [26], collating the existing literature on this topic is essential to understand the efficacy of different interventions in improving eating habits in team sport athletes. In this regard, a previous systematic review [18] analyzed the influence of nutrition education interventions on eating habits, finding most studies (13 out of 16 studies) reported positive changes in dietary behavior post-intervention across athletes competing in twenty-five different individual and team sports. Furthermore, meta-analytic evidence [16] showed most nutrition education interventions $(85.7 \%)$ significantly increased nutrition knowledge in athletes competing in individual and team sports following different nutrition education interventions with face-to-face strategies the most effective intervention modality ( 28 out of 36 studies). Due to the strong relationships that dietary behaviors and nutrition knowledge hold with body composition and physical performance $[9,27,28]$, many studies have examined the effects of nutrition education interventions on body composition [22,29-33] and performance indicators [32,34,35]. Regarding body composition, Nascimento et al. [31] applied nutrition counselling consisting of four consultations separated by 45 to 60 days in adult and adolescent team and individual sport athletes, observing increased lean body mass in both groups after the intervention (pre-intervention $=48.0 \pm 1.2 \mathrm{~kg}$ vs. post-intervention $=49.2 \pm 1.6 \mathrm{~kg} ; p<0.05$ ). Regarding physical performance, Rossi et al. [32] reported improved change-of-direction speed (change $=-0.15 \pm 0.13 \mathrm{~s}$; $p<0.001$ ) and vertical jump height (change $=6.6 \pm 9.4 \mathrm{~cm} ; p<0.001$ ) in collegiate baseball players following a nutrition education intervention involving 90-min information sessions focused on food preparation, nutrients, and healthy eating habits across 12 weeks. Although multiple systematic reviews have explore the effectiveness of education interventions on dietary behaviors and nutrition knowledge $[16,18]$, no systematic reviews have focused explicitly on team sport athletes in this area nor examined the effectiveness of nutrition education interventions on performance indicators in any athlete groups.

Despite the strong interest in conducting nutrition education interventions in team sports, there is currently no scientific consensus for the most effective intervention to apply in practice. Therefore, a systematic review of the literature is essential to generate robust conclusions regarding the effects of different nutrition education interventions in team sport athletes and facilitate their application to practice in specific contexts. Consequently, the aim of this systematic review was to analyze the 
effects of nutrition education interventions on eating habits, nutrition knowledge, body composition, and physical performance in team sport athletes.

\section{Materials and Methods}

This review was carried out following the recommendations and criteria established in the Preferred Reporting Items for Systematic Reviews and Meta-analysis (PRISMA) statement guidelines [36].

\subsection{Search Strategy}

To identify potential studies, a systematic search was performed in the following databases: PubMed/MEDLINE, Web of Science (including all Web of Science Core Collection: Citation Indexes) and SPORTDiscus. The search syntax included the following keywords with relevant Boolean operators inserted: ("education intervention" OR "nutritional intervention" OR "nutrition education") AND ("nutrition knowledge" OR "health knowledge" OR "food knowledge" OR "diet knowledge" OR "dietary behavior" OR "dietary behaviour" OR "dietary intake" OR "dietary assessment" OR "food habit" OR "body composition" OR "weight management" OR "performance" OR "physical performance" OR "athletic performance") AND ("team sport" OR "athlete"). A year restriction was applied for this search (i.e., studies published between 1980 and 2020). Furthermore, the reference lists of included studies were searched, and studies that cited the included studies were located using Google Scholar and checked for their relevance. Two authors (S.S-D. and D.C.) independently screened the title and abstract of each study. Full-text versions of studies that met the inclusion criteria were then screened. Discrepancies regarding inclusion of studies between authors were decided by consensus with a third author (J.R-G.). The search was performed on 21 November 2020.

\subsection{Inclusion Criteria}

Studies meeting the following criteria were included in our review: (1) sample in experimental group was composed solely of team sport athletes; (2) nutrition intervention implemented using educational strategies; (3) instrument(s) used produced quantitative scores pre- and post-intervention; (4) intervention impact was assessed on any of the following outcomes: eating habits, nutrition knowledge, body composition, and/or physical performance indicators; and (5) a full-text version of the study was published in a peer-reviewed journal. In addition, studies that were not written in English, as well as conference abstracts, letters to the editor, errata, narrative reviews, systematic reviews, meta-analyses, or invited commentaries were excluded from our review.

\subsection{Study Coding and Data Extraction}

The following data were extracted from the included studies: (a) authors, year of publication, and study design; (b) sample characteristics (including sample size, sex, age, country, sport modality, and competition level); (c) tool/questionnaire/parameter/test used for each analyzed variable; (d) intervention procedure (modality, frequency, and duration); and (e) major findings (i.e., positive/negative/unchanged effects on eating habits, nutrition knowledge, body composition, and/or physical performance).

\subsection{Methodological Quality Assessment}

To assess the methodological quality of the included studies, a modified version of the Downs and Black checklist was used [37]. In this regard, items 8, 13, and 17 were removed, and the tool was complemented with two additional items (i.e., 9 and 10) from the Academy of Nutrition and Dietetics (AND) quality criteria checklist [38] for greatest relevance to nutrition intervention studies [16]. In the checklist, each question is answered with "yes" if the criteria are satisfied or "no" if the criteria are not satisfied. The answer "yes" was awarded one point for all items (and the answer "no" given zero points) except items 5 and 18, which were scored with a maximum of two points. This approach 
allowed reviewers to differentiate between studies providing two or less validity or reliability measures for the chosen nutrition knowledge instrument and studies that used three or more validity or reliability measures for the chosen nutrition knowledge instrument. Additionally, for studies using single-arm designs in our review, items 14, 15, 21, 22, 23, and 24 were not deemed relevant, so the maximum score for studies using this study design was 22 points with the following scoring criteria adopted: $\leq 11$ points: poor quality; $12-15$ points: fair quality; $16-19$ points: good quality; $20-22$ points: excellent quality). For studies using double-arm designs, the maximum score was 28 points with the following scoring criteria adopted: $\leq 14$ points: poor quality; $15-19$ points: fair quality; $20-25$ points: good quality; 26-28 points: excellent quality) [16,39].

Data extraction and methodological quality assessment were performed by two authors (S.S-D. and J.R-G.) independently, and a third author was consulted (D.D.) to solve any discrepancies via consensus.

\subsection{Search Results}

Figure 1 shows the study retrieval process performed for our review. A total of 456 studies were identified in the initial search, while three additional studies were identified through other sources (i.e., ResearchGate). One hundred and thirty-one study duplicates were eliminated, and 325 studies were screened. Furthermore, 276 studies were excluded based on their titles and/or abstracts. Full-text versions of the remaining 49 studies were assessed for eligibility, with 14 studies meeting the inclusion criteria and being retained in our systematic review [22-26,29,30,32-35,40-42].
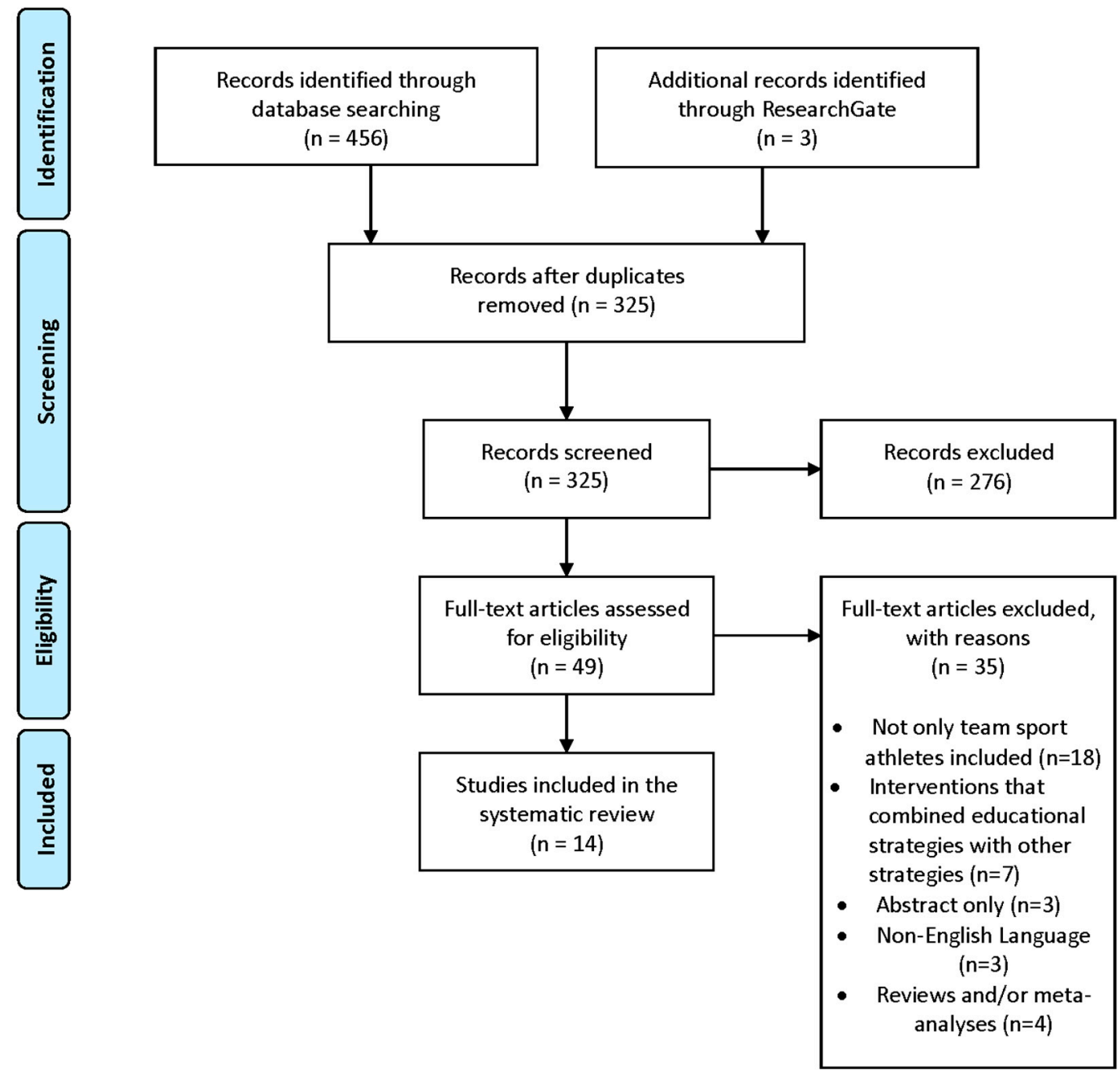

Figure 1. Flow diagram of the study retrieval process. 


\section{Results}

\subsection{Descriptive Characteristics of the Studies}

Tables 1-4 summarize the characteristics of the 14 included studies according to the outcome variables analyzed. In this regard, 10 studies analyzed the impact of nutrition education interventions on eating habits [22-26,29,30,32,33,40], nine studies analyzed nutrition knowledge [22-26,32,40-42], five studies analyzed body composition [22,29,30,32,33], and three studies analyzed physical performance $[32,34,35]$ in team sport athletes. Regarding the experimental design, seven studies used single-arm designs [22,30,32,34,40-42] and seven studies used double-arm designs [23-26,29,33,35]. Moreover, quasi-experimental pre-post and repeated measures designs were used. Included studies examined a total of 683 athletes with five studies examining only male athletes [24,26,30,41,43], six studies examining only female athletes [22,25,29,32,33,40], and three studies examining male and female athletes combined [23,34,35]. Four studies examined volleyball athletes [22,29,33,40], three studies examined soccer athletes [23-25], two studies examined field and ice hockey athletes [41,42], one study examined handball athletes [30], one study examined basketball athletes [32], and three studies examined athletes from different team sports [26,34,35]. Finally, interventions between ten days and two seasons were applied, including from 2 to 12 educational sessions and using several delivery modalities (i.e., face-to-face, game, technological platform, workshop, comic book, and sport nutrition lessons).

\subsection{Methodological Quality Assessment}

Tables 5 and 6 show the methodologic quality of the included studies. In single-arm studies, quality scores ranged from 6 to 16 points, with an average of $11.7 \pm 3.4$ points, while double-arm studies ranged from 13 to 21 points, with an average of $17.4 \pm 2.8$ points. Regarding quality assessment of each study, four studies were categorized as poor quality, seven studies were categorized as fair quality, and three studies were categorized as good quality. 
Table 1. Characteristics and major findings of the studies analyzing the effect of nutrition education interventions on eating habits in team sport athletes.

\begin{tabular}{|c|c|c|c|c|c|c|c|c|c|}
\hline Authors & Year & $\begin{array}{l}\text { Sample } \\
\text { Size }(n)\end{array}$ & $\begin{array}{c}\text { Age } \\
(\mathrm{M} \pm \mathrm{SD} \\
\text { Years })\end{array}$ & Country & Sport & $\begin{array}{l}\text { Competition } \\
\text { Level }\end{array}$ & Tool & $\begin{array}{l}\text { Intervention (Modality, } \\
\text { Frequency and Duration) }\end{array}$ & Major Findings \\
\hline $\begin{array}{l}\text { Abood et al. } \\
{[25]}\end{array}$ & 2004 & $\begin{array}{c}\text { NEI } \\
\text { (15 females) } \\
\text { CG } \\
\text { (15 females) }\end{array}$ & $\begin{array}{c}\text { NEI } \\
(19.6 \pm 1.0) \\
\text { CG }(19.4 \pm 1.2)\end{array}$ & United States & $\begin{array}{c}\text { NEI } \\
\text { Soccer } \\
\text { CG } \\
\text { Swimming }\end{array}$ & $\begin{array}{l}\text { College } \\
\text { division I }\end{array}$ & $\begin{array}{l}\text { 3-day food } \\
\text { record }\end{array}$ & $\begin{array}{l}\text { Nutrition education intervention } \\
\text { NEI } \\
\text { (online, } 8 \text { sessions of } 1 \mathrm{~h}, 8 \\
\text { weeks) } \\
\text { CG } \\
\text { (no treatment) }\end{array}$ & $\begin{array}{l}\text { NEI athletes experienced a } \\
\text { significant increase in } \\
\text { self-efficacy compared to CG. } \\
\text { CG recorded a decrease in } \\
\text { carbohydrate } \\
\text { intake (percentage of total } \\
\text { calories) and fiber. } \\
\text { There was a significant overall } \\
\text { difference in the number of } \\
\text { positive dietary changes } \\
\text { favoring the NEI. }\end{array}$ \\
\hline $\begin{array}{l}\text { Anderson } \\
\text { et al. [29] }\end{array}$ & 2010 & $\begin{array}{c}\text { Feedback } \\
\text { (8 females) } \\
\text { Baseline } \\
\text { (8 females) }\end{array}$ & $\begin{array}{c}\text { Feedback } \\
(20.1 \pm 0.5) \\
\text { Baseline } \\
(19.3 \pm 0.5)\end{array}$ & United States & Volleyball & $\begin{array}{l}\text { NCAA } \\
\text { division II }\end{array}$ & $\begin{array}{l}\text { 3-day food } \\
\text { record }\end{array}$ & $\begin{array}{c}\text { Feedback regarding dietary } \\
\text { intake } \\
\text { Feedback } \\
\text { (F2F at beginning, peak during, } \\
\text { and following each season for } \\
\text { two full seasons). } \\
\text { Baseline } \\
\text { (no treatment) }\end{array}$ & $\begin{array}{l}\text { No changes in total } \\
\text { carbohydrate, protein, and fat } \\
\text { intake between baseline and } \\
\text { feedback across the two } \\
\text { seasons; however, greater } \\
\text { protein intake was recorded } \\
\text { after feedback at beginning of } \\
\text { the season. }\end{array}$ \\
\hline $\begin{array}{l}\text { Daniel et al. } \\
\text { [40] }\end{array}$ & 2016 & 10 females & $17.2 \pm 0.9$ & Brazil & Volleyball & Junior & $\begin{array}{c}\text { Quesitonnaire } \\
\text { of Associação } \\
\text { Brasileira de } \\
\text { Empresas de } \\
\text { Pesquisa }\end{array}$ & $\begin{array}{l}\text { Interdisciplinary Food, Nutrition } \\
\text { and Health Education Program } \\
\text { (F2F, } 8 \text { sessions, } 8 \text { months) }\end{array}$ & $\begin{array}{l}\text { Athletes made dietary changes } \\
\text { during the year increasing } \\
\text { their daily intake of fruits and } \\
\text { vegetables and decreasing } \\
\text { high-energy, low-nutrient food } \\
\text { intake. }\end{array}$ \\
\hline $\begin{array}{l}\text { Elias et al. } \\
\text { [26] }\end{array}$ & 2018 & $\begin{array}{c}\text { NEI } \\
\text { (52 males) } \\
\text { CG } \\
\text { (53 males) }\end{array}$ & $\begin{array}{c}\text { NEI } \\
(18.7 \pm 0.9) \\
\text { CG }(23.3 \pm 3.8)\end{array}$ & Malaysia & $\begin{array}{l}\text { NEI } \\
\text { Field hockey } \\
\text { and soccer } \\
\text { CG } \\
\text { Cricket and } \\
\text { rugby union }\end{array}$ & National & $\begin{array}{c}\text { KAP } \\
\text { questionnaire }\end{array}$ & $\begin{array}{l}\text { Sport education intervention } \\
\text { activities } \\
\text { NEI } \\
\text { (F2F and online, } 7 \times 1.5-\mathrm{h} \\
\text { sessions, } 9 \text { weeks) } \\
\text { CG } \\
\text { (no treatment) }\end{array}$ & $\begin{array}{l}\text { NEI athletes showed higher } \\
\text { mean total energy intake than } \\
\text { CG following the education } \\
\text { intervention. } \\
\text { NEI showed greater increases } \\
\text { in total carbohydrate, protein, } \\
\text { and fat intake than CG } \\
\text { following the education } \\
\text { intervention. }\end{array}$ \\
\hline
\end{tabular}


Table 1. Cont.

\begin{tabular}{|c|c|c|c|c|c|c|c|c|c|}
\hline Authors & Year & $\begin{array}{l}\text { Sample } \\
\text { Size }(n)\end{array}$ & $\begin{array}{c}\text { Age } \\
(\mathrm{M} \pm \mathrm{SD} \\
\text { Years })\end{array}$ & Country & Sport & $\begin{array}{l}\text { Competition } \\
\text { Level }\end{array}$ & Tool & $\begin{array}{l}\text { Intervention (Modality, } \\
\text { Frequency and Duration) }\end{array}$ & Major Findings \\
\hline $\begin{array}{l}\text { Molina-López } \\
\text { et al. [30] }\end{array}$ & 2013 & 14 males & $22.9 \pm 2.7$ & Spain & Handball & Professional & $\begin{array}{l}\text { 3-day food } \\
\text { record }\end{array}$ & $\begin{array}{l}\text { Nutrition education program } \\
\text { (F2F, } 3 \text { phases, } 5 \text { months) }\end{array}$ & $\begin{array}{l}\text { Significant increase in total } \\
\text { energy and macronutrient } \\
\text { intakes following the } \\
\text { intervention. }\end{array}$ \\
\hline $\begin{array}{l}\text { Patton-Lopez } \\
\text { et al. [23] }\end{array}$ & 2018 & $\begin{array}{c}\text { NEI } \\
\text { (153 males } \\
\text { and females) } \\
\text { CG } \\
\text { (64 males and } \\
\text { females) }\end{array}$ & $\begin{array}{c}\text { NEI } \\
(14.9 \pm 0.9) \\
\text { CG }(14.9 \pm 0.9)\end{array}$ & United States & Soccer & High school & $\begin{array}{c}\text { SNK } \\
\text { questionnaire }\end{array}$ & $\begin{array}{c}\text { WAVE education program } \\
\text { NEI } \\
\text { (F2F, } 7 \text { sport nutrition lessons } \\
\text { and } 3 \text { team-building workshops, } \\
1-1.5 \mathrm{~h}, 2 \text { years) } \\
\text { CG } \\
\text { (no treatment) }\end{array}$ & $\begin{array}{l}\text { NEI were three times more } \\
\text { likely to report trying to eat for } \\
\text { performance. } \\
\text { Consumption of lunch } \\
\text { ( } \geq 5 \text {-days per week) did not } \\
\text { change in NEI, but significantly } \\
\text { declined in the CG. }\end{array}$ \\
\hline $\begin{array}{l}\text { Rossi et al. } \\
\text { [32] }\end{array}$ & 2017 & 15 females & $19.3 \pm 1.0$ & United States & Baseball & $\begin{array}{l}\text { College } \\
\text { division I }\end{array}$ & $\begin{array}{l}\text { 3-day food } \\
\text { record }\end{array}$ & $\begin{array}{l}\text { Nutrition education intervention } \\
\text { (F2F, every } 3 \text { weeks, } 12 \text { weeks) }\end{array}$ & $\begin{array}{c}\text { NEI athletes significantly } \\
\text { increased total energy intake, } \\
\text { total carbohydrate intake, and } \\
\text { total protein intake following } \\
\text { the intervention. }\end{array}$ \\
\hline $\begin{array}{l}\text { Valliant et al. } \\
\text { [22] }\end{array}$ & 2012 & $\begin{array}{c}\text { NEI } \\
11 \text { females } \\
\text { CG } \\
11 \text { females }\end{array}$ & $\begin{array}{c}\text { NEI } \\
19.8 \pm 1.0 \\
\text { CG } \\
19.5 \pm 1.0\end{array}$ & United States & Volleyball & $\begin{array}{l}\text { College } \\
\text { division } 1\end{array}$ & $\begin{array}{l}\text { 3-day food } \\
\text { record }\end{array}$ & $\begin{array}{c}\text { Nutrition education } \\
\text { NEI } \\
\text { (F2F, } 4 \text { individual sessions, } \\
4 \text { months) } \\
\text { CG } \\
\text { (no treatment) }\end{array}$ & $\begin{array}{l}\text { NEI athletes significantly } \\
\text { increased total energy, } \\
\text { carbohydrate, and protein } \\
\text { intake following the } \\
\text { intervention. }\end{array}$ \\
\hline $\begin{array}{l}\text { Wenzel et al. } \\
\text { [33] }\end{array}$ & 2012 & $\begin{array}{l}\text { NEI } \\
10 \text { females } \\
\text { CG } \\
29 \text { females }\end{array}$ & $\begin{array}{c}\text { NEI } \\
19.8 \pm \mathrm{NR} \\
\mathrm{CG} \\
19.8 \pm \mathrm{NR}\end{array}$ & United States & Volleyball & $\begin{array}{c}\text { College } \\
\text { division } 1\end{array}$ & $\begin{array}{l}\text { 3-day food } \\
\text { record }\end{array}$ & $\begin{array}{c}\text { Nutrition counselling } \\
\text { NEI } \\
\text { (F2F, once a month, } 4 \text { months) } \\
\text { CG } \\
\text { (no treatment) }\end{array}$ & $\begin{array}{l}\text { Athletes significantly increased } \\
\text { total energy, carbohydrate, and } \\
\text { protein intake following the } \\
\text { intervention. }\end{array}$ \\
\hline $\begin{array}{l}\text { Zeng et al. } \\
\text { [24] }\end{array}$ & 2020 & $\begin{array}{c}\text { NEI } \\
15 \text { males } \\
\text { CG } \\
15 \text { males }\end{array}$ & $\begin{array}{c}\text { NEI } \\
16.7 \pm 1.8 \\
\text { CG } \\
16.8 \pm 1.7\end{array}$ & China & Soccer & Youth top & $\begin{array}{l}\text { 3-day food } \\
\text { record }\end{array}$ & $\begin{array}{l}\text { Nutrition education intervention } \\
\text { NEI } \\
\text { (F2F and comic book, once a } \\
\text { week during } 30 \text { min, } 4 \text { weeks) } \\
\text { CG } \\
\text { (comic book) }\end{array}$ & $\begin{array}{l}\text { No changes were observed in } \\
\text { total carbohydrate, protein, } \\
\text { and fat intake in NEI and CG } \\
\text { following the intervention. }\end{array}$ \\
\hline
\end{tabular}

n: number; M: mean; SD: standard deviation; NEI: nutrition education intervention; CG: control group; F2F: face to face; KAP: nutrition knowledge, attitude and practice; SNK: sport nutrition knowledge; NCAA: National Collegiate Athletic Association. 
Table 2. Characteristics and major findings of the studies analyzing the effect of nutrition education interventions on nutrition knowledge in team sport athletes.

\begin{tabular}{|c|c|c|c|c|c|c|c|c|c|}
\hline Authors & Year & $\begin{array}{l}\text { Sample } \\
\text { Size }(n)\end{array}$ & $\begin{array}{c}\text { Age } \\
(\mathrm{M} \pm \mathrm{SD} \\
\text { Years })\end{array}$ & Country & Sport & $\begin{array}{l}\text { Competition } \\
\text { Level }\end{array}$ & Questionnaire & $\begin{array}{l}\text { Intervention (Modality, } \\
\text { Frequency, and Duration) }\end{array}$ & Major Findings \\
\hline $\begin{array}{l}\text { Abood et al. } \\
{[25]}\end{array}$ & 2004 & $\begin{array}{c}\text { NEI } \\
\text { (15 females) } \\
\text { CG } \\
\text { (15 females) }\end{array}$ & $\begin{array}{c}\text { NEI } \\
(19.6 \pm 1.0) \\
\text { CG }(19.4 \pm 1.2)\end{array}$ & United States & $\begin{array}{c}\text { NEI } \\
\text { Soccer } \\
\text { CG } \\
\text { Swimming }\end{array}$ & $\begin{array}{l}\text { College } \\
\text { division I }\end{array}$ & $\begin{array}{l}\text { Flesch-Kincaid } \\
\text { Grade Level } \\
\text { Index }\end{array}$ & $\begin{array}{l}\text { Nutrition education intervention } \\
\text { NEI } \\
\begin{array}{c}\text { (online, } 8 \times 1 \text {-h sessions, } 8 \text { weeks) } \\
\text { CG } \\
\text { (no treatment) }\end{array}\end{array}$ & $\begin{array}{l}\text { NEI athletes experienced a } \\
\text { significant increase in nutrition } \\
\text { knowledge and self-efficacy } \\
\text { compared to CG. }\end{array}$ \\
\hline $\begin{array}{l}\text { Daniel et al. } \\
\quad[40]\end{array}$ & 2016 & 10 females & $17.2 \pm 0.9$ & Brazil & Volleyball & Junior & $\begin{array}{c}\text { KAP } \\
\text { questionnaire }\end{array}$ & $\begin{array}{l}\text { Interdisciplinary Food, Nutrition } \\
\text { and Health Education Program } \\
\text { (F2F, } 8 \text { sessions, } 8 \text { months) }\end{array}$ & $\begin{array}{c}\text { NE athletes improved their } \\
\text { nutrition knowledge from } \\
57.0 \pm 9.9 \% \text { at baseline to } \\
63.0 \pm 11.8 \% \text { following the } \\
\text { intervention. }\end{array}$ \\
\hline $\begin{array}{l}\text { Elias et al. } \\
{[26]}\end{array}$ & 2018 & $\begin{array}{c}\text { NEI } \\
\text { (52 males) } \\
\text { CG } \\
\text { (53 males) }\end{array}$ & $\begin{array}{c}\text { NEI } \\
(18.7 \pm 0.9) \\
\text { CG }(23.3 \pm 3.8)\end{array}$ & Malaysia & $\begin{array}{l}\text { NEI } \\
\text { Field hockey } \\
\text { and soccer. } \\
\text { CG } \\
\text { Cricket and } \\
\text { rugby union }\end{array}$ & National & $\begin{array}{l}\text { Jürgensen } \\
\text { questionnaire }\end{array}$ & $\begin{array}{l}\text { Sport education intervention } \\
\text { activities } \\
\text { NEI } \\
\text { (F2F and online, } 7 \times 1.5-\mathrm{h} \\
\text { sessions, } 9 \text { weeks) } \\
\text { CG } \\
\text { (no treatment) }\end{array}$ & $\begin{array}{c}\text { NEI athletes showed } \\
\text { significant increases } \\
(-6.21 \pm 2.95 \text { points) in } \\
\text { nutrition knowledge after the } \\
\text { intervention. } \\
\text { Significant decrease } \\
(-2.15 \pm 1.45 \text { points }) \text { in } \\
\text { nutrition knowledge after the } \\
\text { intervention in the CG. }\end{array}$ \\
\hline $\begin{array}{l}\text { Patton-Lopez } \\
\text { et al. [23] }\end{array}$ & 2018 & $\begin{array}{c}\text { NEI } \\
\text { (153 males } \\
\text { and females) } \\
\text { CG } \\
\text { (64 males and } \\
\text { females) }\end{array}$ & $\begin{array}{c}\text { NEI } \\
(14.9 \pm 0.9) \\
\text { CG }(14.9 \pm 0.9)\end{array}$ & United States & Soccer & High school & $\begin{array}{c}\text { SNK } \\
\text { questionnaire }\end{array}$ & $\begin{array}{c}\text { WAVE education program } \\
\text { NEI } \\
\text { (F2F, } 7 \text { sport nutrition lessons } \\
\text { and } 3 \text { team building workshops, } \\
1-1.5 \mathrm{~h}, 2 \text { years) } \\
\text { CG } \\
\text { (no treatment) }\end{array}$ & $\begin{array}{l}\text { NEI athletes significantly } \\
\text { increased their nutrition } \\
\text { knowledge scores from } \\
5.16 \pm 1.80 \text { points at baseline to } \\
6.09 \pm 1.59 \text { points after the } \\
\text { 1-year intervention, while there } \\
\text { were no changes in the CG. }\end{array}$ \\
\hline $\begin{array}{l}\text { Reading } \\
\text { et al. [41] }\end{array}$ & 1999 & 33 males & $14.3 \pm 3.6$ & Canada & Ice hockey & Youth & $\begin{array}{l}\text { Modified } \\
\text { version of } \\
\text { SNAC } \\
\text { questionnaire }\end{array}$ & $\begin{array}{l}\text { Sport Nutrition for the Athletes } \\
\text { of Canada (SNAC) } \\
\text { (F2F, online and lectures, } 4 \times 1 \text {-h } \\
\text { sessions, } 2 \text { weeks) }\end{array}$ & $\begin{array}{l}\text { No changes in nutrition } \\
\text { knowledge following the } \\
\text { intervention. }\end{array}$ \\
\hline $\begin{array}{l}\text { Rossi et al. } \\
\text { [32] }\end{array}$ & 2017 & 15 females & $19.3 \pm 1.0$ & United States & Baseball & $\begin{array}{l}\text { College } \\
\text { division } 1\end{array}$ & $\begin{array}{l}\text { Reilly and } \\
\text { Maughan } \\
\text { sports } \\
\text { nutrition } \\
\text { questionnaire }\end{array}$ & $\begin{array}{l}\text { Nutrition education intervention } \\
\text { (F2F, } 3 \text { times per week, } 12 \text { weeks) }\end{array}$ & $\begin{array}{l}\text { NEI athletes significantly } \\
\text { improved their nutrition } \\
\text { knowledge from } 54.7 \pm 14.3 \% \\
\text { at baseline to } 70.0 \pm 9.4 \% \\
\text { following the intervention. }\end{array}$ \\
\hline
\end{tabular}


Table 2. Cont.

\begin{tabular}{|c|c|c|c|c|c|c|c|c|c|}
\hline Authors & Year & $\begin{array}{l}\text { Sample } \\
\text { Size }(n)\end{array}$ & $\begin{array}{c}\text { Age } \\
\text { (M } \pm \text { SD } \\
\text { Years) }\end{array}$ & Country & Sport & $\begin{array}{l}\text { Competition } \\
\text { Level }\end{array}$ & Questionnaire & $\begin{array}{l}\text { Intervention (Modality, } \\
\text { Frequency, and Duration) }\end{array}$ & Major Findings \\
\hline $\begin{array}{l}\text { Simpson } \\
\text { et al. [42] }\end{array}$ & 2017 & 17 males & $19.0 \pm 0.7$ & New Zealand & Hockey & $\begin{array}{l}\text { International } \\
\text { and national }\end{array}$ & $\begin{array}{l}\text { Zin et al. } \\
\text { questionnaire } \\
\text { of nutritional } \\
\text { knowledge }\end{array}$ & $\begin{array}{l}\text { Nutrition education intervention } \\
\text { (online, once a week, } 6 \text { weeks) }\end{array}$ & $\begin{array}{l}\text { NEI athletes significantly } \\
\text { improved their nutrition } \\
\text { knowledge from } 56.7 \pm 11.4 \% \\
\text { at baseline to } 61.1 \pm 11.5 \% \\
\text { following the intervention. }\end{array}$ \\
\hline $\begin{array}{l}\text { Valliant et al. } \\
\text { [22] }\end{array}$ & 2012 & $\begin{array}{l}\text { NEI } \\
11 \text { females } \\
\text { CG } \\
11 \text { females }\end{array}$ & $\begin{array}{l}\text { NEI } \\
19.8 \pm 1.0 \\
\text { CG } \\
19.5 \pm 1.0\end{array}$ & United States & Volleyball & $\begin{array}{l}\text { College } \\
\text { division } 1\end{array}$ & $\begin{array}{l}\text { Reilly and } \\
\text { Maughan } \\
\text { sports } \\
\text { nutrition } \\
\text { questionnaire }\end{array}$ & $\begin{array}{c}\text { Nutrition education intervention } \\
\text { NEI } \\
\text { (F2F, } 4 \text { individual sessions, } 4 \\
\text { months) } \\
\text { CG } \\
\text { (no treatment) }\end{array}$ & $\begin{array}{l}\text { NEI athletes significantly } \\
\text { increased their nutrition } \\
\text { knowledge scores from } \\
24.7 \pm 5.9 \text { points at baseline to } \\
31.5 \pm 6.1 \text { points following } \\
\text { intervention. }\end{array}$ \\
\hline $\begin{array}{l}\text { Zeng et al. } \\
\text { [24] }\end{array}$ & 2020 & $\begin{array}{c}\text { NEI } \\
15 \text { males } \\
\text { CG } \\
15 \text { males }\end{array}$ & $\begin{array}{c}\text { NEI } \\
16.7 \pm 1.8 \\
\text { CG } \\
16.8 \pm 1.7\end{array}$ & China & Soccer & Youth top & $\begin{array}{c}\text { KAP } \\
\text { questionnaire }\end{array}$ & $\begin{array}{l}\text { Nutrition education intervention } \\
\text { NEI } \\
\text { (F2F and comic book, once a } \\
\text { week during } 30 \text { min, } 4 \text { weeks) } \\
\text { CG } \\
\text { (comic book) }\end{array}$ & $\begin{array}{l}\text { NEI improved general and } \\
\text { sports nutrition knowledges } \\
\text { following the intervention. }\end{array}$ \\
\hline
\end{tabular}

n: number; M: mean; SD: standard deviation; NEI: sport nutrition education intervention; CG: control group; F2F: face to face; KAP: nutrition knowledge, attitude and practice; SNK: sport nutrition knowledge.

Table 3. Characteristics and major findings of the studies analyzing the effect of nutrition education interventions on body composition in team sport athletes

\begin{tabular}{|c|c|c|c|c|c|c|c|c|c|}
\hline Authors & Year & $\begin{array}{l}\text { Sample } \\
\text { Size }(n)\end{array}$ & $\begin{array}{c}\text { Age } \\
(\text { Mean } \pm \text { SD) }\end{array}$ & Country & Sport & $\begin{array}{c}\text { Competition } \\
\text { Level }\end{array}$ & Parameter & $\begin{array}{l}\text { Intervention (Modality, } \\
\text { Frequency, and Duration) }\end{array}$ & Major Findings \\
\hline $\begin{array}{l}\text { Anderson } \\
\text { et al. [29] }\end{array}$ & 2010 & $\begin{array}{l}\text { Feedback } \\
\text { (8 females) } \\
\text { Baseline } \\
\text { (8 females) }\end{array}$ & $\begin{array}{l}\text { Feedback } \\
(20.1 \pm 0.5) \\
\text { Baseline } \\
(19.3 \pm 0.5)\end{array}$ & United States & Volleyball & $\begin{array}{l}\text { NCAA } \\
\text { division II }\end{array}$ & $\begin{array}{l}\text { Body mass } \\
\text { Body fat }(\%)\end{array}$ & $\begin{array}{c}\text { Feedback regarding dietary } \\
\text { intakes } \\
\text { Feedback } \\
\text { (F2F, at beginning, peak } \\
\text { during, and following each } \\
\text { season for two full seasons) } \\
\text { Baseline } \\
\text { (no treatment) }\end{array}$ & $\begin{array}{l}\text { No changes in body mass and } \\
\text { body fat percentage at } \\
\text { beginning, peak, and following } \\
\text { the season between the first } \\
\text { season (no information given) } \\
\text { and the second season } \\
\text { (feedback). }\end{array}$ \\
\hline $\begin{array}{l}\text { Molina-López } \\
\text { et al. [30] }\end{array}$ & 2013 & 14 males & $22.9 \pm 2.7$ & Spain & Handball & Professional & $\begin{array}{c}\text { Body mass } \\
\text { Body mass } \\
\text { index } \\
\text { Body fat }(\%)\end{array}$ & $\begin{array}{c}\text { Nutrition education } \\
\text { program } \\
\text { (F2F, } 3 \text { phases, } 5 \text { months) }\end{array}$ & $\begin{array}{l}\text { NEI athletes showed no } \\
\text { changes in body mass index } \\
\text { and body fat percentage } \\
\text { following the intervention. }\end{array}$ \\
\hline
\end{tabular}


Table 3. Cont

\begin{tabular}{|c|c|c|c|c|c|c|c|c|c|}
\hline Authors & Year & $\begin{array}{l}\text { Sample } \\
\text { Size }(n)\end{array}$ & $\begin{array}{c}\text { Age } \\
(\text { Mean } \pm \text { SD) }\end{array}$ & Country & Sport & $\begin{array}{l}\text { Competition } \\
\text { Level }\end{array}$ & Parameter & $\begin{array}{l}\text { Intervention (Modality, } \\
\text { Frequency, and Duration) }\end{array}$ & Major Findings \\
\hline $\begin{array}{c}\text { Rossi et al. } \\
\text { [32] }\end{array}$ & 2017 & $\begin{array}{c}\text { NEI } \\
\text { (15 females) } \\
\text { CG } \\
\text { (15 females) }\end{array}$ & $\begin{array}{c}\text { NEI } \\
(19.3 \pm 1.0) \\
\text { CG } \\
(19.8 \pm 1.4)\end{array}$ & United States & Baseball & $\begin{array}{l}\text { College } \\
\text { division I }\end{array}$ & $\begin{array}{c}\text { Body mass } \\
\text { Body fat (\%) } \\
\text { Fat free mass } \\
\text { Fat mass }\end{array}$ & $\begin{array}{l}\text { Nutrition education } \\
\text { intervention } \\
\text { NEI } \\
\text { (F2F, every } 3 \text { weeks, } \\
12 \text { weeks) } \\
\text { CG } \\
\text { (no treatment) }\end{array}$ & $\begin{array}{l}\text { NEI athletes significantly } \\
\text { decreased body fat percentage } \\
(-1.4 \pm 2.2 \%) \text { following the } \\
\text { intervention compared to the } \\
\text { CG }(0.5 \pm 2.6 \%) \text {. } \\
\text { NEI athletes significantly } \\
\text { decreased fat mass } \\
(-1.0 \pm 2.0 \%) \text { following the } \\
\text { intervention compared to the } \\
\text { CG }(0.6 \pm 1.4 \%) \text {. }\end{array}$ \\
\hline $\begin{array}{l}\text { Valliant et al. } \\
\text { [22] }\end{array}$ & 2012 & $\begin{array}{c}\text { NEI } \\
11 \text { females } \\
\text { CG } \\
11 \text { females }\end{array}$ & $\begin{array}{c}\text { NEI } \\
19.8 \pm 1.0 \\
\text { CG } \\
19.5 \pm 1.0\end{array}$ & United States & Volleyball & $\begin{array}{l}\text { College } \\
\text { division } 1\end{array}$ & $\begin{array}{c}\text { Fat free mass } \\
(\%) \\
\text { Fat mass (\%) }\end{array}$ & $\begin{array}{c}\text { Nutrition education } \\
\text { NEI } \\
\text { (F2F, } 4 \text { individual sessions, } \\
4 \text { months) } \\
\text { CG } \\
\text { (no treatment) }\end{array}$ & $\begin{array}{l}\text { NEI athletes and CG } \\
\text { significantly decreased body } \\
\text { fat percentage }(\sim 2 \%) \text { and } \\
\text { significantly increased fat-free } \\
\text { mass percentage }(\sim 2 \%) \text {. }\end{array}$ \\
\hline $\begin{array}{l}\text { Wenzel et al. } \\
\text { [33] }\end{array}$ & 2012 & $\begin{array}{c}\text { NEI } \\
10 \text { females } \\
\text { CG } \\
29 \text { females }\end{array}$ & $\begin{array}{c}\text { NEI } \\
19.8 \pm \mathrm{NR} \\
\mathrm{CG} \\
19.8 \pm \mathrm{NR}\end{array}$ & United States & Volleyball & $\begin{array}{l}\text { College } \\
\text { division } 1\end{array}$ & $\begin{array}{l}\text { Body mass } \\
\text { Body fat }(\%)\end{array}$ & $\begin{array}{l}\text { Nutrition counselling } \\
\text { NEI } \\
\text { (F2F, once a month, } \\
4 \text { months) } \\
\text { CG } \\
\text { (no treatment) }\end{array}$ & $\begin{array}{l}\text { NEI athletes significantly } \\
\text { decreased their body fat } \\
\text { percentage }(\sim 2 \%), \text { while CG } \\
\text { did not change. }\end{array}$ \\
\hline
\end{tabular}


Table 4. Characteristics and major findings of the studies analyzing the effect of nutrition education interventions on physical performance in team sport athletes.

\begin{tabular}{|c|c|c|c|c|c|c|c|c|c|}
\hline Authors & Year & $\begin{array}{l}\text { Sample Size } \\
\text { (n) }\end{array}$ & $\begin{array}{c}\text { Age } \\
(\mathrm{M} \pm \mathrm{SD} \\
\text { Years) }\end{array}$ & Country & Sport & $\begin{array}{l}\text { Competition } \\
\text { Level }\end{array}$ & Test & $\begin{array}{l}\text { Intervention (Modality, } \\
\text { Frequency and Duration) }\end{array}$ & Major Findings \\
\hline $\begin{array}{l}\text { Kavouras } \\
\text { et al. [35] }\end{array}$ & 2012 & $\begin{array}{c}\text { NEI } \\
\text { (61 males and } \\
\text { females) } \\
\text { CG } \\
\text { (31 males and } \\
\text { females) }\end{array}$ & $\begin{array}{c}\text { NEI }(14.0 \pm \\
4.8) \\
\text { CG }(13.2 \pm 2.3)\end{array}$ & Greece & $\begin{array}{l}\text { Volleyball and } \\
\text { basketball }\end{array}$ & Youth & $\begin{array}{l}\text { 600-m run } \\
\text { 30-m sprint } \\
\text { Vertical jump } \\
\text { Skill test }\end{array}$ & $\begin{array}{c}\text { Hydration intervention } \\
\text { plan } \\
\text { NEI (F2F, } 3 \text { times per day, } \\
10 \text { days) } \\
\text { CG (no treatment) }\end{array}$ & $\begin{array}{l}\text { NEI athletes significantly } \\
\text { decreased } 600-\text { m running time } \\
(-12.7 \pm 1.5 \%) \text { following the } \\
\text { intervention. }\end{array}$ \\
\hline $\begin{array}{l}\text { Rossi et al. } \\
\text { [32] }\end{array}$ & 2017 & $\begin{array}{c}\text { NEI } \\
\text { (15 females) } \\
\text { CG } \\
\text { (15 females) }\end{array}$ & $\begin{array}{c}\text { NEI } \\
(19.3 \pm 1.0) \\
\text { CG } \\
(19.8 \pm 1.4)\end{array}$ & United States & Baseball & $\begin{array}{l}\text { College } \\
\text { division I }\end{array}$ & $\begin{array}{l}\text { 5-10-5 shuttle run } \\
\text { Vertical jump } \\
\text { Broad jump } \\
\text { Squat } 1 \text { RM }\end{array}$ & $\begin{array}{c}\text { Nutrition education } \\
\text { intervention } \\
\text { NEI } \\
\text { (F2F, every } 3 \text { weeks, } \\
12 \text { weeks) } \\
\text { CG } \\
\text { (no treatment) }\end{array}$ & $\begin{array}{c}\text { NEI athletes and CG } \\
\text { significantly decreased 5-10-5 } \\
\text { shuttle run time }(-0.06 \text { to } \\
-0.15 \mathrm{~s}) \text {, and significantly } \\
\text { increased vertical jump height } \\
(5.1-6.6 \mathrm{~cm}) \text { broad jump distance } \\
(9.9-12.0 \mathrm{~cm}) \text {, and } 1 \mathrm{RM} \text { squat } \\
\text { strength }(20.0-27.7 \mathrm{~kg}) \text {. } \\
\text { No significant }(p>0.05) \\
\text { interactions between NEI and } \\
\text { CG for each performance } \\
\text { variable. }\end{array}$ \\
\hline $\begin{array}{l}\text { Shoemaker } \\
\text { et al. [34] }\end{array}$ & 2019 & $\begin{array}{l}\text { Male } \\
(18) \\
\text { Female } \\
(25)\end{array}$ & $\begin{array}{l}\text { Male } \\
(16.6 \pm 1.1) \\
\text { Female } \\
(16.1 \pm 1.0)\end{array}$ & United States & $\begin{array}{c}\text { School-or } \\
\text { club-sponsored } \\
\text { sports }\end{array}$ & NR & $\begin{array}{l}\text { Vertical jump } \\
\text { Broad jump } \\
\text { Agility test } \\
\text { Push-up force }\end{array}$ & $\begin{array}{l}\text { Sports nutrition curriculum } \\
\text { (online, } 7 \text { sessions, } 8 \text { weeks) }\end{array}$ & $\begin{array}{l}\text { Athletes increased vertical jump } \\
\text { peak power }\left(\sim 13 \mathrm{~W} \cdot \mathrm{kg}^{-1}\right) \text {, broad } \\
\text { jump distance }(\sim 7 \mathrm{~cm}) \text {, and } \\
\text { push-up force }(\sim 27 \mathrm{~N}) \text { following } \\
\text { the intervention. }\end{array}$ \\
\hline
\end{tabular}

n: number; M: mean; SD: standard deviation; NEI: sport nutrition education intervention; CG: control group; F2F: face to face education; RM: repetition maximum; NR: not reported. 
Table 5. Methodologic quality of the included single-arm studies.

\begin{tabular}{|c|c|c|c|c|c|c|c|c|c|c|c|c|c|c|c|c|c|c|c|c|c|c|c|c|c|c|c|}
\hline Study & 1 & 2 & 3 & 4 & 5 & 6 & 7 & 8 & 9 & 10 & 11 & 12 & 13 & 14 & 15 & 16 & 17 & 18 & 19 & 20 & 21 & 22 & 23 & 24 & 25 & 26 & Score $(/ 22)$ \\
\hline Daniel et al. (2016) [40] & 1 & 1 & 0 & 1 & 0 & 1 & 1 & 1 & 0 & 0 & 0 & 0 & & & 0 & 0 & 0 & 0 & 0 & & & & & 0 & 0 & 0 & 6 \\
\hline Molina-López (2013) [30] & 1 & 1 & 1 & 1 & 1 & 1 & 1 & 1 & 0 & 0 & 0 & 0 & & & 1 & 1 & 0 & 1 & 1 & & & & & 0 & 1 & 0 & 12 \\
\hline Reading et al. (1999) [41] & 1 & 1 & 1 & 1 & 1 & 1 & 1 & 1 & 1 & 0 & 1 & 1 & & & 1 & 1 & 1 & 1 & 0 & & & & & 0 & 1 & 0 & 16 \\
\hline Rossi et al. (2017) [32] & 1 & 0 & 1 & 1 & 1 & 1 & 1 & 1 & 1 & 0 & 1 & 1 & & & 1 & 1 & 0 & 1 & 0 & & & & & 0 & 1 & 0 & 14 \\
\hline Shoemaker et al. (2019) [34] & 1 & 1 & 1 & 1 & 1 & 1 & 0 & 1 & 1 & 0 & 0 & 1 & & & 1 & 1 & 0 & 1 & 1 & & & & & 0 & 1 & 0 & 14 \\
\hline Simpson et al. (2017) [42] & 1 & 1 & 0 & 1 & 0 & 1 & 1 & 0 & 1 & 0 & 0 & 0 & & & 1 & 1 & 0 & 0 & 0 & & & & & 0 & 1 & 0 & 9 \\
\hline Valliant et al. (2012) [22] & 1 & 1 & 1 & 0 & 0 & 1 & 1 & 1 & 1 & 1 & 0 & 0 & & & 1 & 1 & 0 & 0 & 0 & & & & & 0 & 1 & 0 & 11 \\
\hline
\end{tabular}

1. Hypothesis stated (/1); 2. Outcome described (/1); 3. Characteristics described (/1); 4. Interventions described (/1); 5. Confounders described (/2); 6. Main findings described (/1); 7. Random variability described for main outcomes $(/ 1) ; 8$. Characteristics of athletes lost to follow-up reported $(/ 1) ; 9$. Actual probability reported $(/ 1) ; 10$. Biases and limitations considered (/1); 11. Representative of population (/1); 12. Participating subjects representative (/1); 13. Attempt to blind subjects (/1); 14. Attempt to blind main outcomes (/1); 15. Data dredging reported $(/ 1) ; 16$. Statistics used appropriate $(/ 1) ; 17$. Compliance with intervention reliable $(/ 1) ; 18$. Measurement tool accurate $(/ 2) ; 19$. Funding reported (/1); 20. Groups recruited from same population (/1); 21. Recruited over same time (/1); 22. Randomization (/1); 23. Intervention assignment concealed (/1); 24. Adjustment for confounding (/1); 25. Athletes lost to follow-up accounted for (/1); 26. Sufficient power (/1).

Table 6. Methodologic quality of the included double-arm studies.

\begin{tabular}{|c|c|c|c|c|c|c|c|c|c|c|c|c|c|c|c|c|c|c|c|c|c|c|c|c|c|c|c|}
\hline Study & 1 & 2 & 3 & 4 & 5 & 6 & 7 & 8 & 9 & 10 & 11 & 12 & 13 & 14 & 15 & 16 & 17 & 18 & 19 & 20 & 21 & 22 & 23 & 24 & 25 & 26 & Score $(/ 28)$ \\
\hline Abood et al. (2004) [25] & 1 & 1 & 1 & 1 & 2 & 0 & 0 & 1 & 0 & 1 & 0 & 0 & 0 & 0 & 1 & 1 & 1 & 2 & 0 & 1 & 1 & 1 & 0 & 1 & 1 & 1 & 19 \\
\hline Anderson (2010) [29] & 1 & 1 & 1 & 1 & 1 & 1 & 1 & 0 & 0 & 0 & 0 & 0 & 0 & 0 & 1 & 1 & 0 & 1 & 1 & 1 & 1 & 0 & 0 & 0 & 0 & 0 & 13 \\
\hline Elias et al. (2018) [26] & 1 & 1 & 1 & 1 & 1 & 1 & 1 & 1 & 1 & 1 & 0 & 0 & 0 & 0 & 1 & 1 & 1 & 2 & 0 & 1 & 1 & 1 & 0 & 0 & 1 & 1 & 20 \\
\hline Kavouras et al. (2012) [35] & 1 & 1 & 1 & 1 & 1 & 1 & 1 & 1 & 0 & 1 & 0 & 0 & 0 & 0 & 1 & 1 & 1 & 2 & 0 & 1 & 1 & 0 & 0 & 0 & 1 & 0 & 17 \\
\hline Patton-Lopez et al. (2018) [23] & 1 & 1 & 1 & 1 & 1 & 1 & 1 & 1 & 1 & 1 & 0 & 0 & 0 & 0 & 1 & 1 & 0 & 1 & 1 & 1 & 1 & 0 & 0 & 0 & 0 & 1 & 17 \\
\hline Wenzel et al. (2012) [33] & 1 & 1 & 1 & 1 & 1 & 1 & 1 & 0 & 0 & 1 & 0 & 1 & 0 & 0 & 1 & 1 & 1 & 2 & 1 & 0 & 0 & 0 & 0 & 0 & 0 & 0 & 15 \\
\hline Zeng et al. (2020) [24] & 1 & 1 & 1 & 1 & 1 & 1 & 1 & 1 & 1 & 1 & 0 & 1 & 0 & 0 & 1 & 1 & 1 & 2 & 1 & 1 & 1 & 1 & 0 & 0 & 1 & 0 & 21 \\
\hline
\end{tabular}

1. Hypothesis stated (/1); 2. Outcome described (/1); 3. Characteristics described (/1); 4. Interventions described (/1); 5. Confounders described (/2); 6. Main findings described (/1); 7. Random variability described for main outcomes (/1); 8 . Characteristics of athletes lost to follow-up reported (/1); 9 . Actual probability reported (/1); 10 . Biases and limitations considered (/1); 11. Representative of population (/1); 12. Participating subjects representative (/1); 13. Attempt to blind subjects (/1); 14. Attempt to blind main outcomes (/1); 15. Data dredging reported (/1); 16. Statistics used appropriate (/1); 17. Compliance with intervention reliable (/1); 18. Measurement tool accurate (/2); 19. Funding reported (/1); 20. Groups recruited from same population (/1); 21. Recruited over same time (/1); 22. Randomization (/1); 23. Intervention assignment concealed (/1); 24. Adjustment for confounding (/1); 25. Athletes lost to follow-up accounted for $(/ 1)$; 26 . Sufficient power $(/ 1)$ 


\section{Discussion}

The aim of our systematic review was to analyze the effects of nutrition education interventions on eating habits, nutrition knowledge, body composition, and physical performance in team sport athletes. The main results showed that implementation of nutrition education interventions consistently induced positive changes in eating habits and nutrition knowledge, as well as maintained or ameliorated body composition. Meanwhile the disparity of results across a limited number of studies exploring the effectiveness of education nutrition interventions implies further research is needed on this topic to draw definitive conclusions. These findings suggest the health and performance of team sport athletes from various backgrounds could benefit from nutrition education interventions as a complementary strategy to training routines.

\subsection{Effects of Nutrition Education Interventions on Eating Habits}

Balanced nutritional intakes combined with regular physical activity is advisable for optimal growth and health [44-46]. Additionally, balanced nutritional intakes are essential to ensure suitable nutrients are available to carry out essential systemic functions in the body, while nutrient deficiencies could influence growth and development as well as negatively affect physical performance in young athletes, and promote greater injury risk in all athletes [47]. Nutrient deficiencies can contribute to the development of certain health problems such as diabetes, obesity, sarcopenia, and osteoporosis [48,49]. In this regard, to improve eating habits by means of appropriate macronutrient and micronutrient intakes is convenient to optimize athlete performance during team sport competition, which could be assisted through nutrition education interventions. Our systematic review collated findings from 13 studies exploring the effects of nutrition education interventions on eating habits in team sport athletes, with ten studies showing total energy, carbohydrate, protein, and fat intakes and eating habits such as daily intake of fruits and vegetables as well as reporting trying to eat for performance were ameliorated in team sport athletes [22,25,26,29-33,40]. Additionally, nutrition education interventions provide team sport athletes with knowledge regarding the nutritional properties of food, which could lead to consumption of varied and balanced diets that provide essential nutrients to enhance performance and recovery. It should also be noted that some major limitations were apparent in one study observing nutrition education interventions to yield no changes in eating habits. In this sense, Patton-Lopez et al. [23] noted athletes in the control group declined the consumption of lunch (consuming lunch on less than five days per week) after the intervention, while athletes in the nutrition education intervention group did not change their consumption (consuming lunch on at least five days per week). NEI athletes were three times more likely to report trying to eat for performance, revealing that these athletes were motivated to learn and improve their diet behaviors and benefit from team-based nutrition interventions. Consequently, our review presents strong evidence demonstrating the effectiveness of using nutrition education interventions to improve eating habits in a diverse range of team sport athletes.

\subsection{Effects of Nutrition Education Interventions on Nutrition Knowledge}

Given greater nutrition knowledge is associated with better eating habits and physical conditioning status [50-52], it is relevant for nutrition specialists to ascertain whether nutrition education interventions enhance nutrition knowledge in team sport athletes. In turn, enhanced nutrition knowledge in team sport athletes may promote adoption of a more balanced diet, which could potentially optimize physical preparedness for competition [53]. In this sense, most studies (eight out of nine studies) in our systematic review demonstrated improvements in nutrition knowledge following nutrition education interventions in team sport athletes. Consequently, it appears that team sport athletes develop an understanding of key nutritional concepts following nutrition education interventions to make informed decisions regarding eating habits suited to their training and competition requirements. Contrarily, the study showing no changes in nutrition knowledge following a nutrition education intervention included the shortest intervention period among included studies 
(i.e., 2 weeks), where 33 Canadian ice hockey athletes completed a sport nutrition workbook in a single-arm design [41]. Consequently, future studies should investigate whether longer nutrition education interventions using various modalities improve nutrition knowledge in ice hockey athletes. Considering the effectiveness of nutrition education interventions on nutrition knowledge across most studies, team sport coaching staff should prescribe additional educational strategies to optimize athlete understanding of nutrition to promote eating behaviors that optimize health and performance across the season.

\subsection{Effects of Nutrition Education Interventions on Body Composition}

Possessing optimal body composition is necessary to perform at a high standard during competition in team sports [28]. Given improved body composition can assist team sport athletes in obtaining better physical conditioning and, consequently, reduce injury risk [47,54], understanding whether nutrition education interventions can enhance body composition in team sport athletes is of interest to coaching staff, athletes, and nutrition specialists alike. All five studies examining the effects of nutrition education interventions on body composition in team sport athletes in our systematic review reported improvements in or maintenance of body composition following the intervention. Three studies showed fat mass to decrease [22,32,33] following nutrition education interventions lasting between 3 and 8 months. In contrast, two studies showed no variations in body composition variables (body mass, body mass index, fat mass, body fat percentage, and sum of skinfolds) following implementation of nutrition education interventions [29,30]. The lack of change in body composition detected in these studies may be attributed to the team sport athletes examined presenting with desired body composition variables at the time of baseline testing given athletes can be required to maintain appropriate physical condition across different seasonal phases to adequately cope with training and competition demands.

\subsection{Effects of Nutrition Education Interventions on Physical Performance}

Team sport athletes should undergo physical conditioning that enables them to meet the physical demands of competition [55] and mitigate the fatigue-related injury risk derived from poor conditions [56]. In this sense, a primary goal of team sport coaching staff is to optimize the physical preparedness of athletes for competition which could be ameliorated with appropriate nutritional intake. Thus, including nutrition education strategies during daily routines could be effective in assisting coaches and athletes in achieving this goal. Our systematic review only retrieved three studies analyzing the effects of nutrition education interventions on physical performance in team sport athletes, with disparity in the reported results due to variations in the characteristics of the athletes examined. In this sense, while some nutrition education strategies improved performance during specific fitness tests (i.e., 600-m running time), no change in performance was observed in other tests (i.e., vertical jump and strength tests). For example, Kavouras et al. [35] observed young volleyball and basketball players to improve 600-m running speed following a hydration intervention plan for 10 days, but no improvements were reported in 30-m linear sprint speed, vertical jump height, and skill performance. Similarly, another study assessing the efficacy of an online sports nutrition curriculum across 8 weeks showed improvements in change-of-direction speed and vertical jump height, with no variations in upper-body strength in male and female team sport athletes [34], while another study demonstrated improvements in change-of-direction speed and vertical jump height in female basketball athletes receiving face-to-face nutrition education for 12 weeks and controls [32]. Considering the mixed results regarding changes in physical performance following nutrition education interventions, further research is needed to build the evidence base for team sport athletes specific to sport modality, competition level, age, and sex to uncover the most appropriate nutrition education intervention to optimize physical performance in different contexts. 


\subsection{Limitations}

This study contains some important limitations that should be considered when interpreting the reported effects of nutrition education interventions on eating habits, nutrition knowledge, body composition, and physical performance in team sport athletes. Firstly, it is difficult to establish a definitive consensus regarding specific nutrition education strategies that are most effective at enhancing eating habits, nutrition knowledge, body composition, and physical performance considering the wide range of athletes examined across studies encompassing different sports, ages, competition levels, and cultures. For these reasons, a meta-analysis was not permissible to undertake. Secondly, the small sample of athletes recruited in some studies should be considered when interpreting findings indicating the effectiveness of nutrition education strategies in team sport athletes across the literature. Thirdly, studies included in our review only analyzed physical performance using physical fitness testing, which does not represent actual in-game performance during team sport competition. Consequently, future studies are encouraged examining the effects of nutrition education interventions on in-game performance variables to provide evidence with greater ecological validity on this topic. Fourthly, 11 out of the 14 studies in our review had poor to fair methodological quality suggesting the methodological approaches of studies need to improve on this topic moving forward. Fifthly, the inclusion of athletes from different sports in the control group in some double-arm studies [25,26] should be considered when interpreting the associated results given the variations in physical attributes and nutritional needs that may exist between athletes from different sports [57]. Finally, body composition was measured with different approaches possessing varied validity and reliability across studies including anthropometry, air displacement plethysmography, and dual-energy x-ray absorptiometry, which should be considered when interpreting the findings presented. Future research should expand on the available body composition variables and explore the effects of nutrition education interventions on other variables such as bone mineral content.

\section{Conclusions}

Nutrition education interventions are an effective strategy to improve or maintain eating habits, nutrition knowledge, and body composition in team sport athletes when applied in addition to regular training routines. However, the effects of nutrition education interventions on physical performance in team sport athletes are not clear given the limited studies and mixed findings on this topic. Additionally, due to the heterogeneity across studies included in our review regarding the characteristics of the athletes examined (i.e., sport modality, competition level, age, and sex) and intervention strategies adopted (i.e., modality, frequency, and duration) it is difficult to establish optimal nutrition education interventions to adopt in specific contexts based on the existing literature.

Author Contributions: S.S.-D., D.C. and J.R.-G. conceived and designed the study; D.C. and J.R.-G. analyzed and interpreted the data; S.S.-D., D.C. and J.R.-G. drafted the manuscript; J.Y. and A.T.S. reviewed and prepared the final version of the manuscript. All authors have read and agreed to the published version of the manuscript.

Funding: The research leading to these results has received funding from "la Caixa" Foundation and Caja de Burgos Foundation, under agreement LCF/PR/PR18/51130008.

Conflicts of Interest: The authors declare no conflict of interest.

\section{References}

1. Hulteen, R.M.; Smith, J.J.; Morgan, P.J.; Barnett, L.M.; Hallal, P.C.; Colyvas, K.; Lubans, D.R. Global participation in sport and leisure-time physical activities: A systematic review and meta-analysis. Prev. Med. 2017, 95, 14-25. [CrossRef]

2. Lera-Lopez, F.; Ollo-Lopez, A.; Rapún, M. Sports spectatorship in Spain: Attendance and consumption. Eur. Sport Manag. Q. 2012, 12, 265-289. [CrossRef]

3. Crompton, J.L.; Lee, S. The economic impact of 30 sports tournaments, festivals, and spectator events in seven U.S. cities. J. Park Recreat. Adm. 2000, 18, 107-216. 
4. Mujika, I. The alphabet of sport science research starts with Q. Int. J. Sports Physiol. Perform. 2013, 8, 465-466. [CrossRef]

5. Impellizzeri, F.M.; Marcora, S.M.; Coutts, A.J. Internal and External Training Load: 15 Years On. Int. J. Sports Physiol. Perform. 2019, 14, 270-273. [CrossRef]

6. Lesinski, M.; Prieske, O.; Helm, N.; Granacher, U. Effects of Soccer Training on Anthropometry, Body Composition, and Physical Fitness during a Soccer Season in Female Elite Young Athletes: A Prospective Cohort Study. Front. Physiol. 2017, 8, 1093. [CrossRef] [PubMed]

7. Raya-González, J.; García-Esteban, S.; Hume, P.; Castillo, D. Effects of gluteal muscles strengthening on lower-limb injuries in male professional handball players: A preliminary study. J. Strength Cond. Res. 2020. [CrossRef] [PubMed]

8. Mujika, I.; Halson, S.; Burke, L.M.; Balagué, G.; Farrow, D. An Integrated, Multifactorial Approach to Periodization for Optimal Performance in Individual and Team Sports. Int. J. Sports Physiol. Perform. 2018, 13, 538-561. [CrossRef] [PubMed]

9. Beck, K.L.; Thomson, J.S.; Swift, R.J.; Von Hurst, P.R. Role of nutrition in performance enhancement and postexercise recovery. Open Access J. Sports Med. 2015, 6, 259-267. [CrossRef]

10. Paoli, A.; Bianco, A.; Grimaldi, K.A. The Ketogenic Diet and Sport. Exerc. Sport Sci. Rev. 2015, 43, $153-162$. [CrossRef]

11. Durkalec-Michalski, K.; Zawieja, E.E.; Zawieja, B.; Jurkowska, D.; Buchowski, M.S.; Jeszka, J. Effects of Low Versus Moderate Glycemic Index Diets on Aerobic Capacity in Endurance Runners: Three-Week Randomized Controlled Crossover Trial. Nutrients 2018, 10, 370. [CrossRef] [PubMed]

12. Kondo, E.; Sagayama, H.; Yamada, Y.; Shiose, K.; Osawa, T.; Motonaga, K.; Ouchi, S.; Kamei, A.; Nakajima, K.; Higaki, Y.; et al. Energy Deficit Required for Rapid Weight Loss in Elite Collegiate Wrestlers. Nutrients 2018, 10, 536. [CrossRef] [PubMed]

13. Castillo, D.; Domínguez, R.; Fernández, A.R.; Raya-González, J. Effects of Caffeine Supplementation on Power Performance in a Flywheel Device: A Randomised, Double-Blind Cross-Over Study. Nutrients 2019, 11, 255. [CrossRef]

14. Rodríguez-Fernández, A.; Castillo, D.; Raya-González, J.; Domínguez, R.; Bailey, S.J. Beetroot juice supplementation increases concentric and eccentric muscle power output. Original investigation. J. Sci. Med. Sport 2020. [CrossRef] [PubMed]

15. Raya-González, J.; Rendo-Urteaga, T.; Domínguez, R.; Castillo, D.; Rodríguez-Fernández, A.; Grgic, J. Acute Effects of Caffeine Supplementation on Movement Velocity in Resistance Exercise: A Systematic Review and Meta-analysis. Sports Med. 2019, 50, 717-729. [CrossRef] [PubMed]

16. Tam, R.; Beck, K.L.; Manore, M.M.; Gifford, J.; Flood, V.M.; O'Connor, H. Effectiveness of Education Interventions Designed to Improve Nutrition Knowledge in Athletes: A Systematic Review. Sports Med. 2019, 49, 1769-1786. [CrossRef] [PubMed]

17. Murimi, M.W.; Kanyi, M.; Mupfudze, T.; Amin, R.; Mbogori, T.; Aldubayan, K. Factors Influencing Efficacy of Nutrition Education Interventions: A Systematic Review. J. Nutr. Educ. Behav. 2017, 49, 142-165.e1. [CrossRef]

18. Bentley, M.R.; Mitchell, N.; Backhouse, S.H. Sports nutrition interventions: A systematic review of behavioural strategies used to promote dietary behaviour change in athletes. Appetite 2020, 150, 104645. [CrossRef]

19. Philippou, E.; Middleton, N.; Pistos, C.; Andreou, E.; Petrou, M. The impact of nutrition education on nutrition knowledge and adherence to the Mediterranean Diet in adolescent competitive swimmers. J. Sci. Med. Sport 2017, 20, 328-332. [CrossRef]

20. Doyle-Lucas, A.F.; Davy, B.M. Development and evaluation of an educational intervention program for pre-professional adolescent ballet dancers: Nutrition for optimal performance. J. Dance Med. Sci. 2011, 15, 65-75.

21. Martín-García, A.; Gómez Díaz, A.; Bradley, P.S.; Morera, F.; Casamichana, D. Quantification of a professional football team's external load using a microcycle structure. J. Strength Cond. Res. 2018, 32, 3511-3518. [CrossRef]

22. Valliant, M.; Emplaincourt, H.P.; Wenzel, R.K.; Garner, B.H. Nutrition Education by a Registered Dietitian Improves Dietary Intake and Nutrition Knowledge of a NCAA Female Volleyball Team. Nutrients 2012, 4, 506-516. [CrossRef] 
23. Patton-Lopez, M.M.; Manore, M.M.; Branscum, A.; Meng, Y.; Wong, S.S. Changes in Sport Nutrition Knowledge, Attitudes/Beliefs and Behaviors Following a Two-Year Sport Nutrition Education and Life-Skills Intervention among High School Soccer Players. Nutrients 2018, 10, 1636. [CrossRef]

24. Zeng, D.; Fang, Z.-L.; Qin, L.; Yu, A.-Q.; Ren, Y.-B.; Xue, B.-Y.; Zhou, X.; Gao, Z.-Y.; Ding, M.; An, N.; et al. Evaluation for the effects of nutritional education on Chinese elite male young soccer players: The application of adjusted dietary balance index (DBI). J. Exerc. Sci. Fit. 2020, 18, 1-6. [CrossRef]

25. Abood, D.A.; Black, D.R.; Birnbaum, R.D. Nutrition Education Intervention for College Female Athletes. J. Nutr. Educ. Behav. 2004, 36, 135-139. [CrossRef]

26. Elias, S.S.; Abu Saad, H.; Taib, M.N.M.; Jamil, Z. Effects of sports nutrition education intervention on sports nutrition knowledge, attitude and practice, and dietary intake of Malaysian team sports athletes. Malays. J. Nutr. 2018, 24, 103-116.

27. Burke, L.M.; Jeukendrup, A.E.; Jones, A.M.; Mooses, M. Contemporary Nutrition Strategies to Optimize Performance in Distance Runners and Race Walkers. Int. J. Sport Nutr. Exerc. Metab. 2019, 29, 117-129. [CrossRef]

28. Esco, M.R.; Fedewa, M.V.; Cicone, Z.S.; Sinelnikov, O.A.; Sekulić, D.; Holmes, C.J. Field-Based Performance Tests Are Related to Body Fat Percentage and Fat-Free Mass, But Not Body Mass Index, in Youth Soccer Players. Sports 2018, 6, 105. [CrossRef]

29. Anderson, D. The Impact of Feedback on Dietary Intake and Body Composition of College Women Volleyball Players Over a Competitive Season. J. Strength Cond. Res. 2010, 24, 2220-2226. [CrossRef]

30. Molina-López, J.; Molina, J.M.; Chirosa, L.J.; Florea, D.; Sáez, L.; Jiménez, J.; Planells, P.; de la Cruz, A.P.; Planells, E. Implementación de un programa de educación nutricional en un equipo de balonmano; consecuencias en estado nutricional. Nutr. Hosp. 2013, 28, 1065-1076.

31. Nascimento, M.V.S.D.; Silva, D.; Ribeiro, S.M.L.; Nunes, M.A.P.; Almeida, M.; Netto, R.S.M. Effect of a Nutritional Intervention in Athlete's Body Composition, Eating Behaviour and Nutritional Knowledge: A Comparison between Adults and Adolescents. Nutrients 2016, 8, 535. [CrossRef]

32. Rossi, F.E.; Landreth, A.; Beam, S.; Jones, T.; Norton, L.; Cholewa, J.M. The Effects of a Sports Nutrition Education Intervention on Nutritional Status, Sport Nutrition Knowledge, Body Composition, and Performance during Off Season Training in NCAA Division I Baseball Players. J. Sports Sci. Med. 2017, 16, 60-68.

33. Wenzel, R.K.; Valliant, M.W.; Chang, Y.; Bomba, A.K.; Lambert, L.G. Dietary Assessment and Education Improves Body Composition and Diet in NCAA Female Volleyball Players. Top. Clin. Nutr. 2012, 27, 67-73. [CrossRef]

34. Shoemaker, M.E.; Gillen, Z.M.; McKay, B.D.; Bohannon, N.A.; Mendez, A.I.; Cramer, J.T. Effects Of An Online Sports Nutrition Curriculum On Biomarkers Of Iron Status In Youth Athletes. Med. Sci. Sports Exerc. 2019, 51, 892. [CrossRef]

35. Kavouras, S.A.; Arnaoutis, G.; Makrillos, M.; Garagouni, C.; Nikolaou, E.; Chira, O.; Ellinikaki, E.; Sidossis, L.S. Educational intervention on water intake improves hydration status and enhances exercise performance in athletic youth. Scand. J. Med. Sci. Sports 2012, 22, 684-689. [CrossRef]

36. Moher, D.; Liberati, A.; Tetzlaff, J.; Altman, D.G. PRISMA group preferred reporting items for systematic reviews and meta-analyses: The PRISMA statement. PLoS Med. 2009, 6. [CrossRef]

37. Downs, S.H.; Black, N. The feasibility of creating a checklist for the assessment of the methodological quality both of randomised and non-randomised studies of health care interventions. J. Epidemiol. Community Health 1998, 52, 377-384. [CrossRef]

38. Academy of Nutrition and Dietetics Evidence Analysis Manual: Steps in the Academy Evidence Analysis Process. Chicago, 2016. ISBN 978-0-88091-429-1. Available online: https://www.andeal.org/vault/2440/web/ files/2016_April_EA_Manual.pdf (accessed on 1 September 2020).

39. Raya-González, J.; Clemente, F.M.; Beato, M.; Castillo, D. Injury Profile of Male and Female Senior and Youth Handball Players: A Systematic Review. Int. J. Environ. Res. Public Health 2020, 17, 3925. [CrossRef]

40. Daniel, N.V.S.; Jürgensen, L.P.; Padovani, R.D.C.; Juzwiak, C.R. Impact of an Interdisciplinary Food, Nutrition and Health Education Program for adolescent Brazilian volleyball players. Revista de Nutrição 2016, 29, 567-577. [CrossRef]

41. Reading, K.J.; McCargar, L.J.; Marriage, B.J. Adolescent and Young Adult Male Hockey Players: Nutrition Knowledge and Education. Can. J. Diet. Pr. Res. 1999, 60, 166-169. 
42. Simpson, A.; Gemming, L.; Baker, D.; Braakhuis, A. Do Image-Assisted Mobile Applications Improve Dietary Habits, Knowledge, and Behaviours in Elite Athletes? A Pilot Study. Sports 2017, 5, 60. [CrossRef]

43. Lacome, M.; Simpson, B.M.; Cholley, Y.; Lambert, P.; Buchheit, M. Small-Sided Games in Elite Soccer: Does One Size Fit All? Int. J. Sports Physiol. Perform. 2018, 13, 568-576. [CrossRef]

44. Iglesias-Gutierrez, E.; García-Roves, P.M.; Pablo, M.; Rodríguez, C.; Braga, S.; García-Zapico, P.; Patterson, Á.M. Food Habits and Nutritional Status Assessment of Adolescent Soccer Players. A Necessary and Accurate Approach. Can. J. Appl. Physiol. 2005, 30, 18-32. [CrossRef]

45. Iglesias-Gutiérrez, E.; García, A.; García-Zapico, P.; Pérez-Landaluce, J.; Patterson, A.M.; García-Rovés, P.M. Is there a relationship between the playing position of soccer players and their food and macronutrient intake? Appl. Physiol. Nutr. Metab. 2012, 37, 225-232. [CrossRef]

46. Fraile-Bermúdez, A.B.; Kortajarena, M.; Zarrazquin, I.; Irazusta, A.; Fernandez-Atutxa, A.; Ruiz-Litago, F.; Yanguas, J.J.; Gil, J. Physical activity and dietary habits related to cardiovascular risk in independent community-living older women. Exp. Gerontol. 2017, 92, 46-51. [CrossRef]

47. Grant, J.A.; Bedi, A.; Kurz, J.; Bancroft, R.; Gagnier, J.J.; Miller, B.S. Ability of Preseason Body Composition and Physical Fitness to Predict the Risk of Injury in Male Collegiate Hockey Players. Sports Heal. A Multidiscip. Approach 2015, 7, 45-51. [CrossRef]

48. Hita-Contreras, F.; Martínez-Amat, A.; Cruz-Díaz, D.; Pérez-López, F.R. Osteosarcopenic obesity and fall prevention strategies. Maturitas 2015, 80, 126-132. [CrossRef]

49. Concha-Cisternas, Y.; Guzmán-Muñoz, E.; Valdés-Badilla, P.; Lira-Cea, C.; Petermann, F.; Celis-Morales, C. Levels of physical activity and excess body weight in university students. Rev. Med. Chil. 2018, 146, 840-849. [CrossRef]

50. Ha, S.A.; Lee, S.Y.; Kim, K.A.; Seo, J.S.; Sohn, C.; Park, H.R.; Kim, K.W. Eating habits, physical activity, nutrition knowledge, and self-efficacy by obesity status in upper-grade elementary school students. Nutr. Res. Pr. 2016, 10, 597-605. [CrossRef] [PubMed]

51. Muderedzwa, T.M.; Matsungo, T.M. Nutritional status, physical activity and associated nutrition knowledge of primary school learners. Nutr. Health 2020, 26, 115-125. [CrossRef] [PubMed]

52. Nikolaïdis, P.T.; Theodoropoulou, E. Relationship between Nutrition Knowledge and Physical Fitness in Semiprofessional Soccer Players. Scientifica 2014, 2014, 180353. [CrossRef] [PubMed]

53. García-Roves, P.M.; Pablo, M.; García-Zapico, P.; Patterson, Á.M.; Iglesias-Gutierrez, E. Nutrient Intake and Food Habits of Soccer Players: Analyzing the Correlates of Eating Practice. Nutrients 2014, 6, 2697-2717. [CrossRef] [PubMed]

54. Havenetidis, K.; Paxinos, T.; Kardaris, D.; Bissas, A. Prognostic potential of body composition indices in detecting risk of musculoskeletal injury in army officer cadet profiles. Physician Sportsmed. 2017, 45, 1-6. [CrossRef] [PubMed]

55. Castillo, D.; Raya-González, J.; Weston, M.; Yanci, J. Distribution of External Load During Acquisition Training Sessions and Match Play of a Professional Soccer Team. J. Strength Cond. Res. 2019. [CrossRef]

56. Carling, C.; McCall, A.; Le Gall, F.; Dupont, G. The impact of short periods of match congestion on injury risk and patterns in an elite football club. Br. J. Sports Med. 2015, 50, 764-768. [CrossRef]

57. Holway, F.E.; Spriet, L.L. Sport-specific nutrition: Practical strategies for team sports. J. Sports Sci. 2011, 29, S115-S125. [CrossRef]

Publisher's Note: MDPI stays neutral with regard to jurisdictional claims in published maps and institutional affiliations.

(C) 2020 by the authors. Licensee MDPI, Basel, Switzerland. This article is an open access article distributed under the terms and conditions of the Creative Commons Attribution (CC BY) license (http://creativecommons.org/licenses/by/4.0/). 